\title{
Evolution of Rotating White Dwarfs in Close Binaries and Diversity of Type Ia Supernovae
}

\author{
Tatsuhiro Uenishi, Ken'ichi Nomoto \\ Department of Astronomy, School of Science, University of Tokyo \\ Hongo 7-3-1, Bunkyo-ku, Tokyo 113-0033, Japan
}

Izumi Hachisu

Department of Earth Science and Astoronomy, College of Arts and Science, University of Tokyo

Komaba 3-8-1, Meguro-ku, Tokyo 153-8902, Japan

Type Ia supernovae are very good, but not perfect, standard candles, because their observed brightness shows a little diversity. The origin of this dibersity needs to be understood for the application to cosmology.

In close binary systems, a white dwarf must be rotating faster and faster as it gains angular momentum from the accretion disk. Its rapid rotation affects its final mass and strucure just before a supernova expolosion. Brightness of supernovae can be changed if mass of their progenitors have some diversity.

We used Hachisu's axysymmetric code to compute the structure of axisymmetric rapidly rotating white dwarfs (Figure 1). Each white dwarf is characterized by its maximum dencity and axis ratio. We assumed that a supernova occurs when the maximum dencity reaches the critical density of $2.0 \times 10^{9} \mathrm{gcm}^{-3}$. Dynamical fragmentation criteria is given as $T / W=0.14$, where $T$ is total rotational energy and $W$ is total gravitational energy.

We assumed that a white dwarf is initially at rest and gains mass and angular momentum by accreting matter of Keplerian rotation. Then, we made linear interporation from nearest four points in data table to get physical values for the next step, which are necessary to determine specific angular momentum of accreting gas. Calculation is stopped when dynamical fragmentation or a supernova occurs.

For both rotation laws, if all angular momentum of accreting matter is added to the white dwarf, the white dwarf reaches the upper edge of the data area, where dynamical fragmentation occurs(Figure 2). It means that Type Ia supernovae may not occur under this condition. However, accreting efficiency of angular momentum may be smaller if the white dwarf blows magnetic wind. If the efficiency is $20 \%$, for example, white dwarfs can reach the critical dencity for supernova explosion(Figure 3).

In this case, mass diversity is about $0.9 M_{\odot}$, which is equivalent to 0.4 magnitude in brightness if we assume brightness is propotional to the mass of ${ }^{56} \mathrm{Ni}$ synthesized in supernova explosion. It is very interesting that a smaller initial mass results in a larger final mass because a smaller white dwarf can accrete more matter and can rotate faster and become heavier. This can explain the dependence of supernova brightness on the galaxy type. 

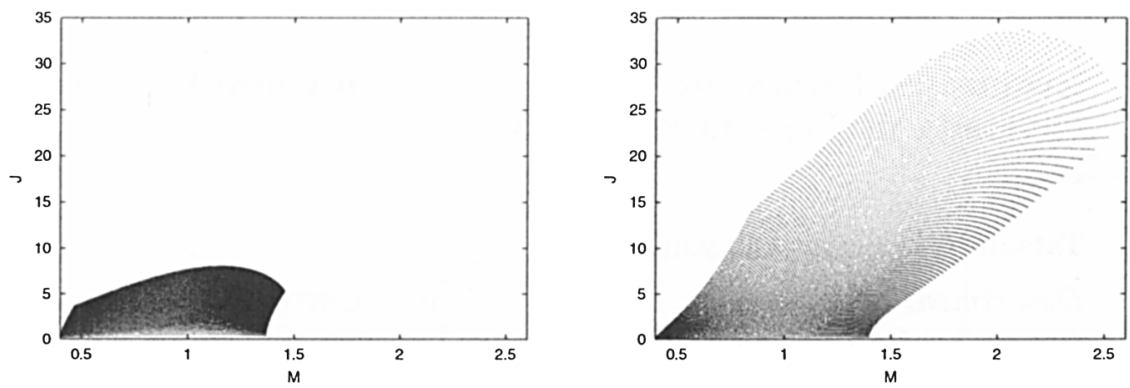

Figure 1. The left and right panels show the data tables for rigid rotation, and j-constant rotation, respectively. Each point means a white dwarf of different angular momentum $\mathrm{J}$ and mass $\mathrm{M}$ (axis ratio and maximum density). Right edge of data area is $\rho_{\max }=2.0 \times 10^{9} \mathrm{gcm}^{-3}$ and upper edge means ratio of surface gravity to centrifugal force is unity (for rigid rotation) or $T / W=0.14$ (for j-constant).
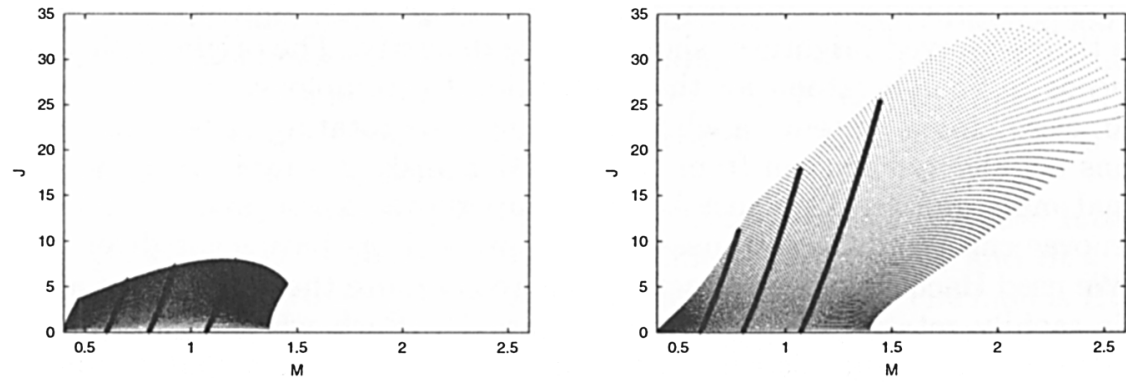

Figure 2. The left panel shows the evolutionary track for white dwarfs initial masses of which are $0.6 M_{\odot}, 0.8 M_{\odot}, 1.07 M_{\odot}$. Efficiecy of angular momentum transport is unity and rotation law is rigid. The right panel shows the same white dwarfs but for the j-constant rotation law.

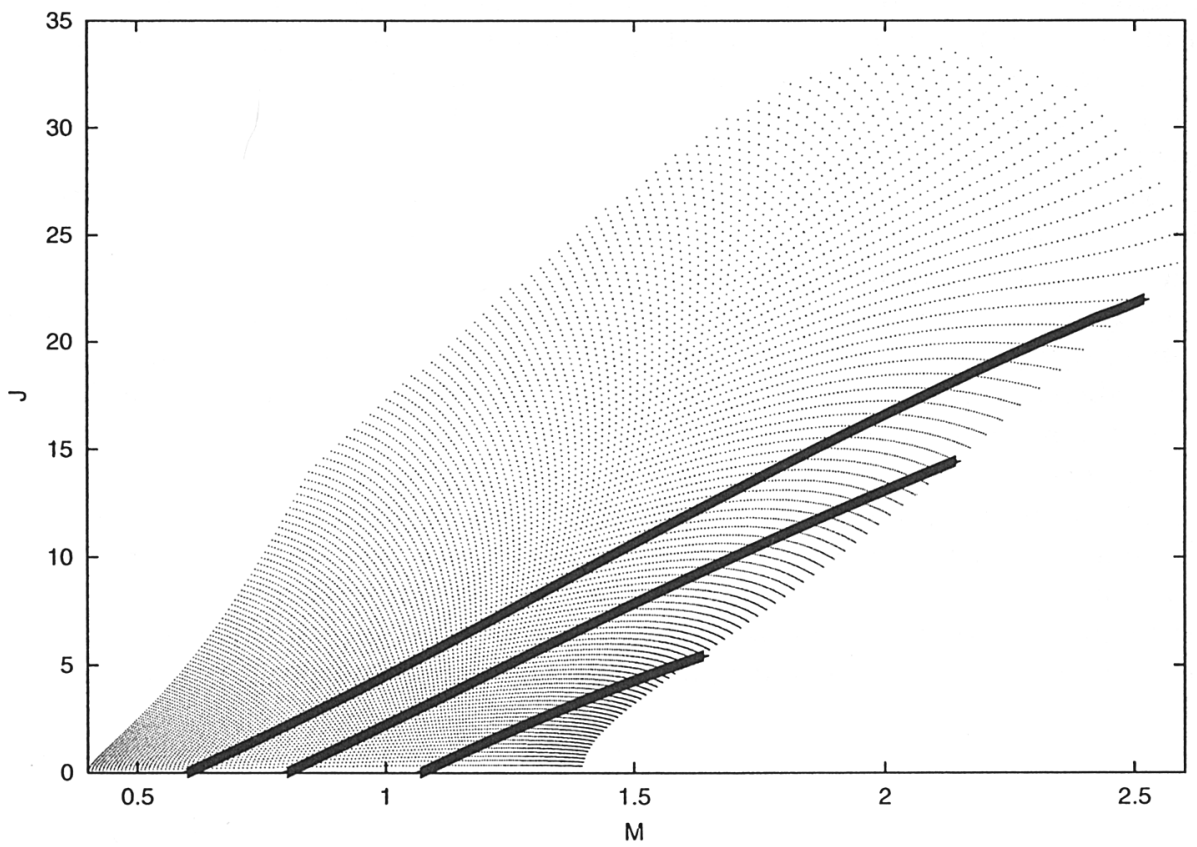

